\title{
Essais
}

Revue interdisciplinaire d'Humanités

$15 \mid 2019$

Jouer l'histoire

\section{Le jeu vidéo « historique » comme objet de recherche}

La représentation vidéoludique de la Grande Guerre en question

José-Louis de Miras

\section{OpenEdition}

\section{Journals}

Édition électronique

URL : http://journals.openedition.org/essais/1371

DOI : $10.4000 /$ essais. 1371

ISSN : 2276-0970

Éditeur

École doctorale Montaigne Humanités

Édition imprimée

Date de publication : 15 octobre 2019

Pagination : 7-21

ISBN : 979-10-97024-07-9

ISSN : $2417-4211$

\section{Référence électronique}

José-Louis de Miras, «Le jeu vidéo « historique » comme objet de recherche », Essais [En ligne], 15 |

2019, mis en ligne le 05 octobre 2020, consulté le 12 octobre 2020. URL : http://

journals.openedition.org/essais/1371; DOI : https://doi.org/10.4000/essais.1371 


\section{Le jeu vidéo " historique » comme objet de recherche: la représentation vidéoludique de la Grande Guerre en question}

Avant-propos

En France, la place du jeu vidéo à l'université n'a eu de cesse d'être débattue alors que sa fulgurante ascension en tant qu'industrie culturelle ne peut plus être dissimulée ${ }^{2}$. Souvent méprisés, considérés comme "dangereux pour la santé de l'enfant ${ }^{3}$ " et risquant " d'abrutir davantage qu'ils n'éveillent l'intelligence ${ }^{4}$ ", les jeux vidéo intriguent la communauté universitaire qui désormais tend à apprécier leur importance économique, culturelle et sociale. Le mouvement d'intérêt actuel envers les jeux vidéo semble en effet s'inscrire dans "l'affirmation du poids économique du secteur ${ }^{5}$ ", mais aussi dans sa

1 Marc Marti, "L'Histoire dans le jeu vidéo, une généalogie narrative problématique ? Le cas de la guerre d'Espagne (1936-1939) et de sa ludicisation ", Science du jeu, 9/2018, 8 juin 2018, p. 2 : https://journals.openedition.org/sdj/1041 (dernière visite le 02/03/2019).

2 Dans son ouvrage publié en 2010, Alexis Blanchet interroge lui-aussi la place du jeu vidéo à l'université, en affirmant que certains "seront peut-être surpris d'apprendre que le jeu vidéo est un objet d'étude dont l'université française s'est emparée depuis quelques années. " Voir, Alexis Blanchet, Des Pixels à Hollywood. Cinéma et jeu vidéo, une histoire économique et culturelle, Houdan, Éditions Pix’n Love, 2010, p. 9.

3 Sébastien Genvo, Introduction aux enjeux artistiques et culturels des jeux vidéo, Paris, l'Harmattan, 2003, p. 26. L'auteur reprend ici la une du grand quotidien populaire anglais, The Sun, "Nintendo a tué mon fils », datant de l'hiver 1992.

4 Ibid., p. 28.

5 Aymeric de Guillomont, "Les jeux dont vous êtes le héros : analyse sémio-actantielle des jeux vidéo en solo ", p. 138-160, in Sébastien Genvo (éd.), Le Game design de jeux vidéo. Approches de l'expression vidéoludique, Paris, l'Harmattan, 2005, p. 140. En 2015, le chiffre d'affaires mondial du jeu vidéo (91,3 milliards de dollars) a dépassé celui du film de divertissement (88,3 milliards de dollars), ce qui semble avoir permis de faciliter l'institutionnalisation du média. Voir également, Emmanuel Forsans, « Marché des jeux vidéo : Chiffre d’affaires mondial 2015, 2016 et 2019 », afv.com, publié le 23 avril 2016 (dernière visite le 02/03/2019). 
légitimation artistique et culturelle. Depuis 1992, la Bibliothèque nationale de France $(\mathrm{BnF})$ reçoit par exemple tous les jeux vidéo distribués en France dans leurs différentes formes éditoriales ${ }^{6}$, tandis que depuis 2003 l'association $\mathrm{MO} 5 . \mathrm{COM}^{7}$ permet de faire le lien entre les amateurs-collectionneurs de jeux vidéo, les acteurs de l'industrie vidéoludique et les institutions, en exposant partout en France les machines et jeux emblématiques qui ont fait l'histoire du média. L'institutionnalisation culturelle du jeu vidéo a véritablement débuté à partir de 2003 avec la mise en place de fonds d'aide publics ${ }^{8}$ et plus particulièrement avec le Fonds d'Aide à l'Édition Multimédia (FAEM) géré par le Centre National du cinéma et de l'image animée (CNC). En complément de ce fonds d'aide, le CNC propose depuis 2008 un crédit d'impôt jeu vidéo ${ }^{9}$ destiné à soutenir et à développer la création française de jeu vidéo ${ }^{10}$.

S'il s'apprête à fêter son cinquantième anniversaire d'existence commerciale $^{11}$, le jeu vidéo est avant tout une industrie en constante évolution, si bien qu'en France son chiffre d'affaires est passé de 1,67 milliard d'euros en $2002^{12}$ à 3,46 milliards d'euros en $2016^{13}$, soit une évolution de $+207 \%$ en 14 ans. Nombreux sont les universitaires et spécialistes du jeu vidéo qui ont retracé l'histoire d'un média naviguant au gré des avancées technologiques, sociales et culturelles. Dans les années 1990, Alain et Frédéric Le Diberder ont à plusieurs

6 La collection de la BnF comptabilise dorénavant plus de 15000 jeux et des centaines de magazines spécialisés : http://bnf.libguides.com/c.php?g=659946\&p=4659785\#17806966 (dernière visite le 02/03/2019).

7 Site de l'association MO5.COM : http://mo5.com/asso/presentation/.

8 Communiqué officiel du CNC rédigé par Caroline Cesbron, chargée des relations presse auprès de la direction générale du CNC de 2001 à 2007, rendu public le 26 septembre 2003 : http://www.culture.gouv.fr/culture/actualites/communiq/aillagon/aide-multimedia.html (dernière visite le 02/03/2019).

9 En octobre 2005, le nouveau ministre de la Culture et de la Communication, Renaud Donnedieu de Vabres, et le nouveau ministre délégué à l'Industrie, François Loos, ont confirmé la future mise en place d'un crédit d'impôt jeu vidéo visant à compléter le FAEM et destiné à "soutenir et à développer la création française de jeu vidéo " : http://www.edubourse.com/finance/actualites.php?actu=23988 (dernière visite le 02/03/2019).

10 CNC. "Aides à la création de jeux vidéo ", bilan 2015 publié en octobre 2016, p. 2 : http://www.cnc.fr/c/document_library/get_file?uuid=7d775751-e86f-46eb-9543ec819802e82f\&groupId=18 (dernière visite le 02/03/2019)

11 Il est généralement admis que Pong (Atari), sorti en 1972, est le premier jeu vidéo à avoir été commercialisé au monde. Â ce titre, et au même titre que la première projection publique des frères Lumière pour le cinéma en 1895, nombreux sont à prendre l'année 1972 comme point de départ de l'industrie du jeu vidéo.

12 Ce chiffre d'affaires est en progression de $21 \%$ par rapport à 2001. Voir, Serge Dupuy Fromy, "Les jeu vidéo dans la société française : des années 1970 au début des années 2000 ", thèse de doctorat en histoire contemporaine, sous la direction de André Ancreve, Université ParisEst, 2012, publiée le 9 septembre 2013, p. 317 : https://tel.archives-ouvertes.fr/tel-00859676/ document (dernière visite le 02/03/2019).

13 L'Essentiel du jeu vidéo, février 2017, sell.fr, p. 8 : http://www.sell.fr/sites/default/files/EJV_ Bilan\%20Mache\%202016_SELL_Fev\%2017\%20BD.pdf (dernière visite le 02/03/2019). 
reprises abordé le phénomène vidéoludique dans son ensemble (son histoire, son économie, sa sociologie), en analysant le contenu des premiers programmes vidéoludiques, leurs effets sur la création, dans la famille et la société ${ }^{14}$. Dans les années 2000, certains spécialistes du jeu vidéo ont publié des ouvrages dédiés aux " petites histoires " du média, à l'image de l'Ultime histoire du jeu vidéo proposé par le journaliste américain Steven L. Kent en $2001^{15}$, tandis que d'autres se sont appuyés sur cette histoire pour envisager le jeu vidéo comme industrie culturelle et artistique. En 2004, dans sa sixième édition de sa Saga des Jeux Vidéo sortie en 1997, Daniel Ichbiah s'est donné pour objectif de conserver la mémoire du jeu vidéo et d'interroger la place de l'artiste dans l'industrie vidéoludique ${ }^{16}$. La même année, l'universitaire Stéphane Natkin propose de transformer le jeu vidéo en tant qu'exploit technique en œuvre audiovisuelle interactive, l'auteur considérant que les jeux vidéo constituent un paradigme des médias du XXI siècle. Pour soutenir sa thèse, Natkin explique qu'en plus d'être le seul média interactif à avoir trouvé une grande stabilité, le jeu vidéo est le premier média à expérimenter la convergence des médias classiques, de l'Internet et des moyens de communication interpersonnelle ${ }^{17}$. Citons enfin les travaux d'Alexis Blanchet, novateurs et originaux en ce qu'ils retracent l'histoire commune du cinéma et du jeu vidéo, leur convergence et l'impact que leur relation a eu sur le fonctionnement des industries du loisir et sur les processus de production des fictions contemporaines ${ }^{18}$.

L'histoire du jeu vidéo est un axe de recherche fort intéressant d'un point de vue économique, industriel et culturel, mais ce n'est pas le seul possible. Quatre autres approches semblent en effet se dessiner ${ }^{19}$ : la psychologie et les sciences cognitives se concentrent sur les effets du jeu vidéo compris « d'un point de vue psychologique, social ou psychosocial ${ }^{20}$ "; les sciences de l'information et de la communication étudient « la construction de l'identité dans les univers

14 Les auteurs iront jusqu’à proposer un guide destiné aux parents pour " gérer les jeux vidéo dans la famille ». Voir, Alain et Frédéric Le Diberder, L'Univers des jeux vidéo, Paris, La Découverte, 1998, p. 222. À noter que l'ouvrage des frères Le Diberder est en réalité une édition refondue et actualisée de Qui a peur des jeux vidéo, Paris, La Découverte, 1993.

15 Notre traduction du titre. Voir, Steven L. Kent, The Ultimate History of Video Games, New York, Three Rivers Press, 2001. Voir également, Rusel Demaria, Johnny L. Wilson, Highscore: The Illustrated History of Electronic Games, New York, Osbrone McGraw Hill, 2002.

16 L'auteur explique notamment que dans les années 1970, Atari interdisait aux artistes d'indiquer leur nom dans le générique de fin. Voir, Daniel Ichbiah, La Saga des jeux vidéo. De Pong à Lara Croft, Paris, Vuibert, 2004, p. 5.

17 L'auteur ajoute que les concepteurs de jeu ont réussi à développer une forme d'écriture interactive qui révolutionne les principes de narration. Voir, Stéphane Natkin, Jeux vidéo et médias du XXI siècle. Quels modèles pour les nouveaux loisirs numériques? (2004), Paris, Vuibert, 2010, p. 2.

18 Voir, Alexis Blanchet, Des Pixels à Hollywood, op. cit. Voir également du même auteur, Les Jeux vidéo au cinéma, Paris, Armand Colin, 2012.

19 À ce sujet, voir Alexis Blanchet, ibid., p. 11.

20 Ibid. 
virtuels et persistants ${ }^{21}$ "; les études esthétiques et cinématographiques s'interrogent sur les enjeux de l'hybridation entre le cinéma et le jeu vidéo, mais analysent aussi les transformations qu'apporte l'interactivité aux notions narratologiques telles que le récit interactif; enfin, la sociologie s'intéresse aux rapports qu'entretient le jeu vidéo avec la culture populaire, mais aussi, comme le relève très justement Alexis Blanchet, "aux tenants idéologiques de certaines productions ${ }^{22}$ ". C'est sous un angle différent, mais non moins complémentaire, que ce numéro de la revue Essais souhaite se positionner. Il s'agit en effet ici de contribuer à un domaine d'étude en pleine expansion et qui envisage le jeu vidéo comme un objet de représentation, au même titre que le cinéma, ainsi que d'étudier l'impact et les enjeux que l'interactivité vidéoludique a sur l'histoire et sur ses représentations dans les jeux vidéo. L'approche du présent numéro envisage donc le jeu vidéo au carrefour de l'histoire et de son rapport au monde qui nous entoure. En ce sens, elle se rapproche du domaine de recherche anglo-saxon des games studies, initié dans les années 1990 et qui vise à distinguer le jeu vidéo comme objet d'étude en analysant par l'interdisciplinarité une grande variété de phénomènes vidéoludiques. Les jeux vidéo ne sont dès lors plus seulement un phénomène de société, ils sont " le carrefour essentiel d'une redéfinition de notre rapport au monde du récit en images ${ }^{23}$ ". Il semble ainsi légitime d'interroger la relation qu'entretient le jeu vidéo avec le réel, qu'il s'agisse de la représentation vidéoludique de l'histoire ou bien, par exemple, de celle des sociétés d'une époque donnée, dans des mondes virtuels consommés par des millions d'utilisateurs.

Comme le cinéma avant lui, le jeu vidéo s'est à plusieurs reprises intéressé à représenter des périodes historiques précises, mais à la différence du septième art, les expériences vidéoludiques ajoutent un pouvoir jusqu’à présent absent des films : celui d'intervenir dans la représentation, de jouer l'histoire en la rendant interactive et ludique ${ }^{24}$. En autorisant le joueur à intervenir dans la fiction historique, le jeu vidéo ludicise le récit historique, le transforme et interpelle notre vision du passé ; il la rend palpable et accessible tout en lui conférant une dimension ludique. La ludicisation de l'histoire, telle que nous l'entendons ici, est une approche interdisciplinaire nouvelle en ce qu'elle vise à mettre en lumière les effets des usages du passé dans les jeux vidéo ${ }^{25}$. Contrairement au

21 Ibid.

22 Ibid.

23 Erwan Higuinen, Charles Tesson, "Cinéphiles et ludophiles ", Cahiers du cinéma, hors série, septembre 2002, p. 4.

24 Le cinéma interactif propose lui-aussi d'intervenir dans le récit pour modifier le déroulement de l'histoire à partir d'alternatives proposées. Ces œuvres audiovisuelles interactives se distinguent toutefois des jeux vidéo en ce qu'elles n'ont pas pour objectif de manipuler les personnages à l'écran, mais seulement de prendre des choix pour eux, là où les jeux vidéo de guerre demandent aux joueurs de prendre part à la guerre en contrôlant des soldats virtuels.

25 Le terme "ludicisation » est souvent privilégié en sciences du jeu en ce qu'il ne renvoie pas 
terme serious game qui se réfere généralement aux jeux destinés à l'apprentissage $^{26}$ et à la ludification (ou gamification) qui se rapporte à l'application du jeu dans un contexte non ludique ${ }^{27}$, la ludicisation transforme une situation en jeu. Il s'agit donc ici de nous intéresser à la transformation du récit historique en expérience vidéoludique et aux enjeux de cette ludicisation. Comme le relève très justement Marc Marti, la « narration fictionnelle possède avant tout un but esthétique et/ou ludique alors que la narration historique se veut cognitive, transmettant un savoir au service de la science ${ }^{28} »$. La question ici est alors de se demander si le jeu vidéo modifie ou non notre vision du passé et de l'histoire. Autrement dit, il s'agit d'envisager la place de la narration vidéoludique historique selon les modalités relevées par M. Marti.

Longtemps restreints par les capacités techniques des premiers dispositifs vidéoludiques, les jeux vidéo sont dorénavant capables de reproduire fidèlement des décors, objets et personnages de façon à ce que les joueurs puissent les identifier aisément. Les rapports qu'entretient le jeu vidéo avec l'histoire, depuis le début des années 1980 avec la sortie de Castle Wolfenstein (Muse Software, 1981) ${ }^{29}$, ont dès lors été accentués au fil du temps et constituent de fait un objet d'étude inédit en ce qu'ils témoignent de la ludicisation du récit historique qui peut, comme le relève M. Marti, " être ludicisé de plusieurs façons et donner lieu à des fictions ${ }^{30}$ ". De l'Antiquité à nos jours, en passant par le Moyen Âge et l'époque moderne, toutes les périodes ont été représentées par les jeux vidéo, " enclins à reproduire l'histoire de façon fantaisiste ou minutieuse ${ }^{31} »$. Parmi les réalisations recensées par Thomas Rabino en 2013, on remarque que le conflit le plus représenté est celui de la Seconde Guerre

à l'idée que l'on puisse "faire " le jeu, comme c'est le cas avec le terme "ludification ", mais plutôt à l'idée que l'on puisse transformer une situation en jeu. Nous préférons donc ici utiliser le terme "ludicisation » en ce qu'il transforme le récit historique en jeu.

26 Marc Prensky, Digital game-based learning, New York, McGraw-Hill, 2001.

27 Introduite au début des années 2010, l'approche de la gamification est aujourd'hui appliquée dans des domaines aussi variés que le marketing, la santé et l'éduction. À ce sujet, voir, Sebastian Deterding, Dan Dixon, Rilla Khaled et Lennart Nacke, " From game design elements to gamefulness: defining gamification ", p. 9-15, in Proceedings of the 15th International Academic MindTrek Conference: Envisioning Future Media Environments, New York, ACM, 2011. Voir également, Baptiste Monterrat, Élise Lavoué, Sébastien George et Michel Desmarais, «Les effets d’une ludification adaptative sur l'engagement des apprenants ", p. 51-74, in Sciences et Technologie de l'Information et de la Communication pour l'Éducation et la Formation, ATIEF, vol. 24, nº 1, 2007.

28 Marc Marti, «L'Histoire dans le jeu vidéo, une généalogie narrative problématique ? Le cas de la guerre d'Espagne (1936-1939) et de sa ludicisation ", op. cit., p. 2.

29 Sorti sur PC, arcade et consoles, Castle Wolfenstein proposait d'incarner un prisonnier du Reich et demandait au joueur de tuer le plus de SS possible.

30 Marc Marti, «L'Histoire dans le jeu vidéo, une généalogie narrative problématique ? Le cas de la guerre d'Espagne (1936-1939) et de sa ludicisation », op. cit., p. 1.

31 Thomas Rabino, "Jeux vidéo et Histoire ", p. 110-116, Le Débat, 2013/5, n 177, p. 112 : https ://www.cairn.info/revue-le-debat-2013-5-p-110.html (dernière visite le 02/03/2019). 
mondiale qui regroupait au début des années 2010 pas moins de 178 créations. Quant aux grands événements historiques sous-représentés, l'auteur mentionne les jeux prenant pour cadre le Japon féodal (70 jeux), la Grande Guerre (35 jeux) et la guerre de Sécession $(27 \text { jeux })^{32}$. L'une des principales raisons de cet engouement pour la Seconde Guerre mondiale, en plus des stratégies commerciales et des goûts du public, est liée à l'origine géographique des studios de jeux vidéo ${ }^{33}$ : puissances en conflit pendant la Seconde Guerre mondiale, par opposition à la Première Guerre mondiale, les États-Unis et le Japon dominent l'industrie vidéoludique depuis longtemps, avec des studios et des constructeurs emblématiques tels qu'Activision Blizzard, Electronic Arts (EA), Nintendo, Sony et Microsoft ${ }^{34}$. On peut donc dire sans trop exagérer que la Seconde Guerre mondiale reste encore aujourd'hui le théâtre d'affrontements par excellence pour quiconque souhaite développer une production vidéoludique d'envergure ${ }^{35}$. Plus encore, si l'on s'intéresse de plus près aux séries vidéoludiques phares des années 1990-2000 (Medal of Honor, Call of Duty, Battlefield), on remarque assez fortement l'omniprésence de l'armée américaine engagée sur différents théâtres d'opérations après l'attaque de Pearl Harbor. C'est que le jeu vidéo historique, de la même manière que le film de fiction historique, se charge souvent " d'une idéologie et de représentations du passé qui reflètent l'imaginaire collectif ou le roman national cher à des pays comme les États-Unis, où se situe l'un des marchés les plus développés $^{36} »$. Autrement dit, l'histoire dans le jeu vidéo constitue une remarquable toile de fond que l'industrie vidéoludique exploite et utilise comme argument commercial destiné au grand public, là où certaines productions indépendantes se veulent plus fidèles aux faits historiques.

Bien qu'absente ${ }^{37}$, ou du moins souvent écartée au profit d'autres conflits moins statiques comme la Seconde Guerre mondiale et la Guerre froide, la Première Guerre mondiale a été adaptée en différents genres vidéoludiques : en simulateurs de vol avec Red Baron (Dynamix, Sierra, 1990), Wings of Glory (EA, 1995) et plus récemment avec Air Conflicts : Secret Wars (bitCom-

32 Ibid., p. 112-113.

33 Constat partagé par Thomas Rabino, ibid., p. 113.

34 Ubisoft, société française de développement de jeux vidéo, demeure quant à elle l'une des seules exceptions.

35 En septembre et novembre 2018, les studios Activision Blizzard et Electronic Arts ont une nouvelle fois confirmé cette dominance en commercialisant Call of Duty WWII (19 septembre 2018) et Battlefield V (20 novembre 2018), la lettre «V» qui succède au titre d'Electronic Arts faisant référence au geste de la main réalisé par Winston Churchill.

36 Thomas Rabino, "Jeux vidéo et Histoire ", op. cit., p. 110-116.

37 Voir à ce sujet, Julien Lalu, "Représenter la Première Guerre mondiale dans les jeux vidéo : entre absence et uchronie ", centenaire.org, publié le 13 mai 2014 : http://centenaire.org/fr/ espace-scientifique/societe/representer-la-premiere-guerre-mondiale-dans-les-jeux-video-entre (dernière visite le 02/03/2019). 
poser Games, 2011) ${ }^{38}$; en jeux de stratégie et wargames en temps réel ou au tour par tour avec History Line 1914-1918 (Blue Byte Software, 1992) et, entre autres, Commander : The Great War (Slitherine Software, 2012) ; ainsi qu'en jeux de tir à la première personne, dit First-Person Shooter, dont certains utilisent la Première Guerre mondiale comme fond historique destiné à accueillir des fictions uchroniques, à l'image de Necrovision (505 Games, 2009), jeu qui transforme les troupes allemandes en armée de zombies. C'est que le jeu vidéo reste avant toute autre chose une industrie du divertissement et qu'à ce titre, il transforme l'histoire et en propose de nouvelles représentations qui lui sont tantôt fidèles, tantôt infidèles. La faible représentation de 14/18 dans les jeux vidéo peut également se traduire par les difficultés liées à la représentation d'une guerre de position où chaque avancée demandait du temps, là où le game design des jeux vidéo de guerre grand public préconise l'affrontement direct et donc très souvent l'action, ce que les historiens Julien Lalu ${ }^{39}$, Chris Kempshall ${ }^{40}$, Thomas Rabinot ${ }^{41}$ et Adam Chapman ${ }^{42}$ ont à plusieurs reprises relevé. De fait, l'interactivité vidéoludique détricote l'histoire, y apporte des modifications plus ou moins importantes et la surcharge parfois d'action afin de la rendre jouable et ludique pour le plus grand plaisir du public. Il existe donc une réelle dichotomie entre les faits historiques et la façon dont ils sont ludicisés par le jeu vidéo, l'industrie vidéoludique ne se positionnant pas souvent entre ces deux alternatives.

Alors que les années 2000 et 2010 nous ont habitués aux guerres virtuelles futuristes, avec des jeux comme Battlefield 4 (EA, 2013), Call of Duty: Advanced Warfare et Infinite Warfare (Activision, 2014 et 2016), Electronic Arts décide en 2016 de cesser cette compétition qui l'oppose à Activision pour conquérir un nouveau champ de bataille, la Grande Guerre, avec Battlefield 1. Choisir le " numéro un » pour le cinquième opus de sa série principale n'est pas un fait anodin : d'une part, le titre d'EA se veut comme un retour aux sources d'une série basée sur des guerres réelles; d'autres parts, ce numéro renvoie à celui de la Première Guerre mondiale. Avec Battlefield 1, la mode de la guerre réaliste est donc revenue - finis les soldats améliorés aux armes ultra-modernisées et

38 Les avions ne sont pas les seuls engins à avoir été adaptés en simulateurs. On peut citer par exemple le jeu Naval Campaigns: Jutland (HPS Simulations, 2002) qui met en scène la plus grande bataille de la Première Guerre mondiale, opposant la Royal Navy britannique et la Kaiserliche en mer du Nord.

39 Julien Lalu, «Représenter la Première Guerre mondiale dans les jeux vidéo : entre absence et uchronie ", op. cit.

40 Chris Kempshall, The First World War in Computer Games, Basingstoke, Hampshire, Palgrave Pivot, 2015.

41 Thomas Rabino, "Jeux vidéo et Histoire ", op. cit.

42 Adam Chapman, "It's Hard to Play in the Trenches: World War I, Collective Memory and Videogames ", Game Studies, 16-2, décembre 2016 : http://gamestudies.org/1602/articles/ chapman (dernière visite le 02/03/2019). 
place au gaz moutarde et aux fusils à verrous modélisés à partir d'archives ${ }^{43}$ - à tel point qu'Activision répondra à l'appel l'année suivante en commercialisant Call of Duty WWII, centré cette fois sur la Seconde Guerre mondiale. Face à la masse de violence que constituent les jeux de guerre actuels, Ubisoft propose quant à lui de commercialiser en 2014 Soldats Inconnus : Mémoires de la Grande Guerre, une création vidéoludique qui s'apparente davantage à une bande dessinée interactive qu'à un jeu vidéo de guerre. L'origine de cette BD interactive développée en partenariat avec la Mission du Centenaire remonte à 2010, lorsque Yoan Fanise, directeur du contenu, apporta un tas de lettres et de documents historiques qu'il avait hérités de sa grand-mère. La lecture des lettres de son arrière-grand-père au front communiqua une forte émotion aux développeurs, car selon eux, " il y avait un contraste entre les documents militaires qui étaient très factuels, très froids $»^{44}$. Voulant sortir de la logique manichéenne du jeu de guerre où l'on incarne des soldats français et américains face à aux Allemands, le titre d'Ubisoft confisque ici les armes pour, « raconter comment la guerre a affecté [...] des gens ordinaires qu'on a sortis de chez eux pour aller faire la guerre et qui ont connu l'horreur ${ }^{45}$. Soldats Inconnus utilise donc dans un premier temps l'archive de famille pour construire le récit. À cet élément structurel s'ajoute un univers graphique proche de la bande dessinée, qui permet selon les développeurs de "rendre le jeu le plus accessible possible et de toucher un public plus large que celui des jeunes [et c'est] aussi une manière différente de traiter de la guerre dans les jeux vidéo $»^{46}$. La commercialisation de ces jeux durant le Centenaire de la Grande Guerre n'est donc pas le fruit du hasard, et c'est pourquoi ce numéro s'y consacre principalement : si Soldats Inconnus a été développé en partenariat avec la Mission du Centenaire, association dévouée à la commémoration de cette période historique, c'est précisément parce qu'il a été intégré dans un travail de réflexion dont le but est de parler des faits historiques autrement ${ }^{47}$. Quant à Electronic Arts et son jeu

43 Très tôt l'archive a été utilisée dans les jeux vidéo, dans un premier temps pour pallier l'incapacité des dispositifs vidéoludiques à créer des scènes cinématiques réalistes, puis dans un second temps comme élément factuel à partir duquel les développeurs s'appuient pour reproduire les décors, costumes, objets et personnages de l'époque représentée. La fonction de l'archive et l'objet de son utilisation dans les jeux vidéo peuvent aussi être questionnés, en ce que l'archive peut apporter un certain réalisme supplémentaire au récit fictionnel, permettre de le contextualiser, d'apporter des informations historiques additionnelles au joueur - dans une certaine mesure, de le documenter - voire, au contraire, d'être modifiée pour manipuler l'histoire dans le but de servir la fiction.

44 Propos recueillis par Antoine Flandrin, "Soldats inconnus" est un jeu vidéo sur la Grande Guerre, mais ce n'est pas un jeu de guerre ", lemonde.fr, publié le 19 mai 2014 : http://www. lemonde.fr/pixels/article/2014/05/19/soldats-inconnus-est-un-jeu-video-sur-la-grande-guerremais-ce-n-est-pas-un-jeu-de-guerre_4421313_4408996.html (dernière visite le 02/03/2019).

45 Ibid.

46 Ibid.

47 De façon générale, les développeurs de jeux vidéo ne cherchent pas tant la fidélité historique que 
Battlefield 1, on peut y voir une stratégie opportuniste visant à se démarquer de son concurrent direct.

Deux usages du passé peuvent dès lors être délimités : le premier usage fait de l'histoire un décor, une façade qui permet, comme au cinéma, d'entourer la structure fictionnelle "d'un halo d'authenticité » ${ }^{48}$. Le monde fictif se dote ici d'un voile historique qui permet de jouer dans le passé et de convaincre d'une certaine manière le joueur de la véridicité de ce que nous raconte le jeu. Les éléments historiques, qui peuvent se présenter à l'écran sous diverses formes (insertion brute d'archives, reconstitution totale ou partielle, voire hybride), peuvent également structurer le récit, en faisant guise, par exemple, de transition narrative entre deux séquences de jeu ${ }^{49}$. Toutefois, si les jeux vidéo s'emparent de l'histoire pour alimenter la fiction, ils peuvent par la même occasion en déformer le contenu, ces productions ne supposant pas de respecter nécessairement le récit historique ${ }^{50}$. L'interactivité, inhérente à la narration vidéoludique, fait effectivement s'éloigner très vite le joueur du récit historique, pour l'engager davantage dans une posture ludique. Les jeux de guerre comme Battlefield peuvent dès lors être vus comme des espaces des possibles, fictionnels, dans lesquels les éléments historiques peuvent être manipulés et parfois détournés, au détriment d'une dimension ludo-pédagogique.

Le deuxième usage consiste à aller plus loin que la simple utilisation de l'histoire comme décor ; bien que répondant à une problématique du divertissement, le jeu vidéo peut être utilisé comme un outil pédagogique, voire commémoratif, poussant historiens, enseignants et chercheurs à s'emparer du sujet pour questionner, parfois avec virulence, la représentation de l'histoire

la crédibilité. À ce sujet, voir l'interview de Maxime Durant, historien chez Ubisoft Montréal : "Être historien pour le jeu Assassin's Creed", radio-canada.ca, publiée le 4 juin 2018 : https:// ici.radio-canada.ca/premiere/emissions/gravel-le-matin/segments/entrevue/74813/historienubisoft-assassin-creed-jeu-video (dernière visite le 02/03/2019).

48 Laurent Véray, Les Images d'archives face à l'Histoire. De la conservation à la création, Chasseneuildu-Poitou, Scérén, CNDP-CRDP, 2011, p. 127.

49 Qu'il s'agisse d'un narrateur intra ou extra-diégétique, du briefing d'un supérieur ou des mémoires d'un soldat, les images d'archives furent souvent alimentées d'une explication orale dans les jeux vidéo des années 1990 et 2000, ce qui permettait d'une part de donner sens au montage et d'autres parts de réaliser une transition narrative entre deux séquences de jeu. De fait, l'image d'archive est ici le moyen de présenter les événements que le joueur va vivre, mais aussi les résultats de son action.

50 Pour paraphraser Marc Ferro, on pourrait dire qu'à l'instar du réalisateur de fictions historiques, le développeur de jeux historiques " sélectionne dans l'Histoire les faits et les traits qui nourrissent sa démonstration, et il laisse de côté les autres, sans avoir à justifier ou à légitimer son choix. Ainsi il se fait plaisir à ceux qui partagent sa foi, et qui constituent son "public". Que la cause ainsi défendue soit largement partagée, et le business tout comme le prestige du [développeur] y trouvent leur compte. Pas nécessairement l'analyse historique, c'est-à-dire l'intelligibilité des phénomènes. "Voir, Marc Ferro, Cinéma et Histoire (1977), Paris, Gallimard, 1993, p. 220-221. 
dans les jeux vidéo. La sortie, au cours du Centenaire de la Grande Guerre, de Soldats Inconnus et Battlefield 1, mais aussi de Verdun 1914-1918 (Blackmill Games, M2H, 2015), a indubitablement attisé l'intérêt des praticiens de l'histoire pour ces fictions historiques interactives dont le réalisme graphique devient de plus en plus saisissant. La Première Guerre mondiale est à ce titre un excellent support pour questionner l'utilisation de l'histoire par le jeu vidéo et inversement, l'utilisation du jeu vidéo par les historiens, enseignants et chercheurs. Autrement dit, la question ici est de savoir si un jeu sur la Première Guerre mondiale peut à la fois être un divertissement et une leçon d'histoire, entendu par là qu'il incite la communauté scientifique et éducative à s'emparer de ces nouveaux objets pour les questionner, voire les déposséder de leur dimension ludique pour n'en extraire que la partie historique ${ }^{51}$.

Avec Soldats Inconnus, Battlefield 1 ou encore Verdun 1914-1918, les développeurs de jeux de guerre tendent à montrer qu'en plus d'une dimension interactive et ludique, le jeu vidéo peut également être doté d'une dimension pédagogique. En autorisant le joueur à devenir acteur de l'histoire dans des représentations virtuelles mettant en scène les différents théâtres d'opérations qui ont façonné le visage du monde, le jeu vidéo questionne l'histoire. En utilisant des images d'archives dans ces récits interactifs, comme ce fut le cas dans Soldats Inconnus : Mémoires de la Grande Guerre; en faisant appel à des historiens pour conseiller et apporter leur expertise ; et en essayant d'être plus ou moins fidèle aux faits qui ont eu lieu il y a de ça cent ans, tout en évitant de perdre la dimension ludique du média, le jeu vidéo n'est-il pas aujourd'hui un moyen, comme le cinéma de fiction avant lui, de représenter l'histoire, d'en apporter une nouvelle dimension? Mais ces jeux de guerre sont-ils pour autant des jeux sur la guerre, des simulations historiques dont le but premier serait d'éduquer le public ? Force est de constater que sur ce point les avis divergent : tandis que les développeurs de jeux comme Electronic Arts et Ubisoft assument faire des raccourcis historiques pour que le divertissement reste prioritaire, certaines personnalités politiques s'insurgent quant aux aberrations anachroniques présentes dans des produits grandement consommés par les adolescents ${ }^{52}$. D’autres encore, comme les historiens Jean-Clément Martin

51 Jonathan Ore, «Battlefield 1: Can a video game about WWI be both entertaining and a history lesson? First-person shooter could spark wider interest in the Great War ", CBC News, publié le 16 novembre $2016:$ http://www.cbc.ca/news/entertainment/battlefield-1-history-1.3821500 (dernière visite le 02/03/2019).

52 On peut citer l'exemple très médiatique de Jean-Luc Mélenchon qui a vu dans Assassin's Creed Unity (Ubisoft, 2014), jeu vidéo dont l'action se déroule dans le Paris de 1789, une propagande et un « dénigrement de la grande Révolution ». À ce sujet, voir Mathilde Siraud, "Jean-Luc Mélenchon dénonce la "propagande" d'Assassin's Creed Unity ", lefigaro.fr, publié le 13 novembre 2015 : http://www.lefigaro.fr/politique/le-scan/citations/2014/11/13/2500220141113ARTFIG00300-un-responsable-du-parti-de-gauche-denonce-la-propagande-dassassin-s-creed-unity.php (dernière visite le 02/03/2019). 
et Laurent Turcot, s'interrogent sur l'attitude que l'historien doit adopter devant un jeu vidéo historique, en tant que conseiller historique engagé par les studios de jeux vidéo, mais également sur la place du jeu vidéo dans l'enseignement de l'histoire ${ }^{53}$. Face à ces nombreuses interrogations, plusieurs professeurs de collège, de lycée et de l'enseignement supérieur ont décidé de prendre en main les outils du numérique pour analyser, de leur propre initiative, la représentation du passé dans les jeux vidéo. Sont ainsi apparus sur la plateforme de vidéo YouTube des chaînes et podcasts tels que JVH : Jeux vidéo et Histoire (Romain Vincent) ${ }^{54}$, History Creed (Arte Creative) ${ }^{55}$ ou encore Histoire en jeux (William Brou) ${ }^{56}$. Chacun tente dès lors, dans un format qui lui est propre, d'étudier les différents aspects de ces représentations vidéoludiques de l'histoire et d'apporter une dimension pédagogique nouvelle aux fictions vidéoludiques historiques dites grand public. Autrement dit, d'envisager le jeu vidéo comme un nouveau matériau à partir duquel l'histoire et ses représentations pourraient être étudiées.

Lieu de rencontre où se côtoient les pensées d'universitaires, professeurs de collèges et lycées, ainsi que d'historiens ayant officié en tant que conseillers historiques lors de la conception de certains jeux vidéo, ce numéro n'a pas pour dessein de répondre d'une seule voix aux interrogations que soulève l'association de l'histoire, "dont la quintessence se situe dans l'objectivité, et [du] jeu vidéo, qui repose sur les actions du joueur, donc sur une subjectivité appelant un cadre fictionnel ${ }^{57}$ ". Au contraire, il vise à mettre en lumière la pluralité des points de vue de chercheuses et chercheurs venus de différents horizons ; les cadres théoriques empruntent donc ici aux études sur l'histoire, à l'étude du game design, aux études cinématographiques et audiovisuelles ainsi qu'à la narratologie, afin de comprendre le fonctionnement de ces jeux historiques et ainsi mettre en lumière leur dimension pédagogique, didactique et représentationnelle, dans une perspective de compréhension des enjeux de la relation histoire/jeu vidéo qui ne s'arrête pas au jugement de valeur et au fact checking. Cette approche plurielle nous invite in fine à envisager le jeu vidéo comme un objet de représentation à partir duquel de nouvelles pratiques émergent, dont celle qui fait l'objet du présent numéro, à savoir l'appropriation du divertissement vidéoludique comme vecteur de recherche sur l'histoire. Le volume se

53 Voir, Jean-Clément Martin, Laurent Turcot, « Histoire et jeu vidéo », p. 225-230, in Écrire l'Histoire, 16, CNRS Éditions, 2016.

54 Chaîne YouTube JVH : Jeux vidéo et Histoire : https://www.youtube.com/user/JNSretro/ featured (dernière visite le 02/03/2019).

55 Programme History Creed: https://www.youtube.com/playlist?list=PLAS2c81AJ7dS8ny15Ac WTUBQ-inGhdgSy (dernière visite le 02/03/2019).

56 Chaîne YouTube Histoire en jeux : https://www.youtube.com/channel/UCRThjUeXnxL_ BKUhqJv5XBg (dernière visite le 02/03/2019).

57 Thomas Rabino, "Jeux vidéo et Histoire ", op. cit., p. 113 : https://www.cairn.info/revue-ledebat-2013-5-p-110.html (dernière visite le 02/03/2019). 
décompose ainsi en thématiques qui examinent d'un côté les différents enjeux de la ludicisation historique (au niveau industriel, médiatique, culturel, historique et pédagogique) et reflètent de l'autre la diversité des approches ayant contribué à sa parution.

Ce numéro s'ouvre avec deux articles qui interrogent la représentation de l'histoire dans les nouveaux médias interactifs, à savoir le jeu vidéo et le webdocumentaire. L'article de Vincent Boutonnet et David Lefrançois examine la représentation de l'histoire dans la série vidéoludique Assassin's Creed. Bien que ce premier essai s'écarte de l'analyse de jeux spécifiques à la Première Guerre mondiale, il constitue une première approche essentielle pour comprendre les enjeux de la ludicisation historique. Les auteurs, spécialistes de la didactique des sciences humaines et sociales, proposent une analyse transversale qui décrit dans un premier temps l'arc métanarratif qui lie les différents épisodes de la série - à savoir une lutte éternelle entre Templiers et Assassins pour la destinée de l'humanité -, puis envisage la façon dont les joueurs peuvent négocier le sens de ces multiples récits polychroniques. Boutonnet et Lefrançois suggèrent que la représentation de l'histoire dans les jeux vidéo est à la fois réaliste et trompeuse, à la fois rigoureuse et fictive, et par conséquent, pourrait être un authentique outil didactique destiné à l'apprentissage de certains faits historiques. L'essai suivant introduit quant à lui la question de la représentation de la Première Guerre mondiale dans ces nouveaux médias, en abordant notamment l'épineuse question de la forme et de la définition du webdocumentaire à l'épreuve de notre conception actuelle de l'histoire et au prisme de sa relation avec le jeu vidéo. Spécialiste des images cinématographiques dans leur rapport à l'histoire, Clément Puget propose une réflexion personnelle basée sur son analyse d'Apocalypse-10 destins et interroge l'éventualité d'un rapprochement entre webdocumentaire, jeu interactif et science historique entendue comme savoir historique et pratique historienne.

Le duo d'articles suivant offre une réponse pragmatique aux questions soulevées dans les précédents essais, en ce qu'ils émanent d'enseignants ayant développé des mécaniques ludo-pédagogiques en introduisant le jeu vidéo en milieu scolaire. Romain Vincent et William Brou prennent ainsi appui sur leur propre expérience pour brosser le portrait du jeu vidéo comme outil qui se doit d'être encadré par l'enseignant. Dans son article, Romain Vincent étudie le dispositif pédagogique interne au jeu d'Ubisoft, Soldats Inconnus, et sa pratique enseignante en milieu scolaire. S'appuyant sur les travaux de Gilles Brougère, qui questionnent les rapports du jeu et de l'apprentissage ${ }^{58}$, l'auteur propose d'analyser cette bande dessinée interactive comme un dispositif ludique hybride entre un jeu vidéo de divertissement et un logiciel au but éducatif explicite. Son analyse, accompagnée de témoignages d'enseignants 
organisateurs de séances pédagogiques basées sur Soldats Inconnus, dessine le portrait du jeu vidéo en classe et des règles instaurées par l'enseignant pour que les élèves puissent apprendre en jouant. Ces séances ludo-pédagogiques développent ainsi un nouveau gameplay, c'est-à-dire de nouvelles règles (possibilités et limites), qui ne dépendent plus du game design du jeu vidéo, mais du cadre dessiné par l'enseignant lors de ces séances. Si le jeu vidéo ludicise l'histoire, l'enseignement le pondère dès lors de sérieux. Cette nouvelle posture enseignante, inhérente à l'étude du jeu vidéo en milieu scolaire et universitaire, se confirme dans l'article de William Brou. L'auteur y développe une réflexion critique sur sa propre utilisation de Battlefield 1 en cours pour enseigner la Première Guerre mondiale, dans des séances spéciales validées par l'Inspection académique, car s'inscrivant dans le parcours d'Éducation aux Médias et à l'Information de l'Éducation Nationale (EMI). S'appuyant sur la grille d'analyse développée par Yvan Hochet dans son article "Jeu vidéo et enseignement de l'Histoire et de la Géographie " ${ }^{59}$, Brou estime que l'approche d'un fait historique par le jeu vidéo permet de stimuler tous les élèves, y compris ceux en situation de décrochage scolaire. L'auteur suggère toutefois que cette stimulation soit maîtrisée, en plaçant par exemple les élèves en position d'observateurs et non d'acteurs, dans des situations où ils peuvent donner la mort par leurs actions. Pour que Battlefield 1 puisse être un support d'enseignement comme un autre, Brou a développé un travail critique porté sur l'étude des images, du gameplay et du scénario de la première séquence du jeu d'Electronic Arts, tout en questionnant le contexte de production et de réception du FPS historique. L'auteur en conclut que le jeu vidéo produit un discours sur le passé à partir duquel il est possible de penser les nouvelles représentations de l'histoire.

Le quatuor d'articles suivant s'écarte de l'analyse ludo-pédagogique pour interroger les enjeux de l'expression vidéoludique de l'histoire, questionnant par conséquent l'ambivalence qui a trait à la ludicisation historique à l'échelle de la production et du game design. Les auteur.e.s proposent ainsi une réflexion sur les contraintes liées aux jeux vidéo historiques qui doivent à la fois veiller à présenter une image du passé qui tend vers l'authenticité, tout en conservant une dimension ludique qui leur est inhérente. Dans le premier article, Antoine Maillard propose d'explorer les enjeux de l'expression vidéoludique de l'histoire, en axant son propos sur le travail des développeurs des jeux historiques. L'auteur y questionne notamment les conditions de la représentation du conflit, à l'échelle de la production, dans deux jeux diamétralement opposés : la simulation Verdun 1914-1918 et la bande dessinée interactive Soldats Inconnus. Dans cet essai, Maillard met en lumière les différents types de sources que les développeurs utilisent pour recréer virtuellement la Grande

59 Yvan Hochet, "Jeux vidéo et enseignement de l'Histoire et de la Géographie ", Les Jeux Vidéo comme objet de recherche, Paris, Éditions Questions théoriques, 2012, p. 123-134. 
Guerre et qui influencent le développement des jeux. Il y interroge aussi la façon dont ces derniers emploient ces divers documents (photographies, témoignages, lettres, etc.) ainsi que le discours qu'ils adoptent sur la mémoire de 14/18. L'auteur en conclut que si ces jeux adoptent deux visions différentes de l'histoire - Verdun met les combats au centre de l'expérience, là où Soldats Inconnus narre les souffrances de la guerre - ils s'orientent tous deux davantage du côté de l'histotainement, qui désigne l'histoire convoquée uniquement pour le divertissement, la transformant par conséquent en produit culturel présent au cinéma, à la télévision, dans la littérature, en bande dessinée et dans les jeux vidéo. Le deuxième article de ce quatuor thématique poursuit la réflexion en observant plus en détail les tensions entre le cadre ludique et le cadre historique à l'échelle des propriétés et des caractéristiques formelles spécifiques au média vidéoludique. Alexane Couturier propose d'observer les mécaniques de jeu associées aux jeux d'aventure et aux jeux de tir à la première personne, en analysant le game design de Soldats Inconnus et Battlefield 1. L'auteure avance ainsi l'idée que chaque genre vidéoludique implique différentes mécaniques de jeux, différents objectifs, et in fine des représentations multiples d'un même événement historique. À la différence de l'essai précédent, qui envisageait la capacité du médium vidéoludique de représenter avec plus ou moins de fidélité des faits historiques, celui de Couturier éloigne au fur et à mesure son propos de ces considérations pour envisager la manière dont le jeu vidéo, en fonction des spécificités du média, met en relation le joueur avec le passé et en quoi il présente une approche de l'histoire différente des autres médias. L'article de Nicolas Patin semble quant à lui répondre à l'appel lancé par Alexane Couturier, en ce qu'il considère Battlefield 1 comme une expérience de jeu historique tout à fait satisfaisante, indépendamment de ses objectifs commerciaux. Historien spécialiste de la Grande Guerre dans son versant allemand, Patin propose ici une analyse de la représentation vidéoludique du caractère international de la guerre, des relations entre soldats et des différentes formes de violence, dans Battlefield 1. Considérant absurde le fait d'essayer d'appliquer au titre d'Electronic Arts « une sorte de filtrage radical du véritable et du véridique ", l'auteur analyse la façon dont ce jeu fait l'écho de certaines tendances de l'historiographie contemporaine et dépeint certains faits avec une grande maestria, tout en étant contraint de créer des aberrations historiques inhérentes au gameplay d'un jeu de divertissement. L'article de Cyril Lacheze et Marion Weckerle, qui vient clore ce quatrième axe thématique, présente un historique critique des jeux consacrés à la Première Guerre mondiale depuis 1975. En mettant en avant un corpus de 153 jeux, Lacheze et Weckerle actualisent l'histoire du jeu historique centré sur 14/18, bien loin des 35 jeux annoncés par Thomas Rabino en $2013^{60}$. Si les auteur.e.s

60 Notons toutefois que Thomas Rabino se focalise sur ce qu'il considère être les jeux les plus perti- 
assument faire l'inventaire des jeux estampillés Great War, c'est pour mieux mettre en avant le pluralisme des genres vidéoludiques qui se sont intéressés à cette période historique, le tout au prisme des évolutions technologiques et des améliorations des dispositifs vidéoludiques.

L'appel lancé par Nicolas Patin à l'attention des historiens afin d'analyser le jeu vidéo à la fois dans son rapport aux faits historiques et comme produit culturel sur la Grande Guerre, trouve sa résonance dans notre entretien avec Alexandre Lafon, directeur adjoint à la Mission du Centenaire de la Première Guerre mondiale, qui a officié en tant que conseiller historique et pédagogique pour Soldats Inconnus : Mémoires de la Grande Guerre. Dans ce dernier essai, nous avons souhaité mettre en avant le travail d'un historien lors du développement d'un jeu vidéo historique dont l'objectif était d'intéresser les jeunes générations à l'idée de la Première Guerre mondiale, en adaptant le jeu vidéo en outil ludo-pédagogique par la rencontre entre un pédagogue-historien et un studio de jeu vidéo français. Basée sur l'expérience personnelle de Lafon, cette rencontre donne lieu à une discussion qui se veut être l'écho des différents essais du présent numéro, en ce qu'elle en offre un tour d'horizon, dessinant ainsi les traits et les contours de la relation de plus en plus importante entre l'industrie vidéoludique et la recherche universitaire, entre les développeurs que nous proposons d'appeler ludéastes et les historiens ; entre les jeux et leur application en milieu scolaire et in fine, entre le jeu vidéo et l'histoire.

José-Louis de Miras

Université Bordeaux Montaigne jose-louis.de-miras@u-bordeaux-montaigne.fr

nents. De même, si l'on regarde de plus près la liste de jeux recensés par la Mission du Centenaire, on remarque là aussi qu'une sélection a été réalisée, l'organisme commémoratif n'indiquant qu'une quarantaine de jeux, là où l'article de Lacheze et Weckerle en annonce quatre fois plus. 